TITLE:

\title{
Liquid/liquid dynamic phase separation induced by a focused laser
}

$\operatorname{AUTHOR}(S)$ :

Mukai, SA; Magome, N; Kitahata, H; Yoshikawa, K

\section{CITATION:}

Mukai, SA ... [et al]. Liquid/liquid dynamic phase separation induced by a focused laser. APPLIED PHYSICS LETTERS 2003, 83(13): 2557-2559

\section{ISSUE DATE:}

2003-09-29

URL:

http://hdl.handle.net/2433/49851

\section{RIGHT:}

Copyright 2003 American Institute of Physics. This article may be downloaded for personal use only. Any other use requires prior permission of the author and the American Institute of Physics. 


\title{
Liquid/liquid dynamic phase separation induced by a focused laser
}

\author{
Sada-Atsu Mukai, ${ }^{a}$ Nobuyuki Magome, Hiroyuki Kitahata, and Kenichi Yoshikawa ${ }^{\text {b) }}$ \\ Department of Physics, Graduate School of Science, Kyoto University and CREST, Kyoto 606-8502, Japan
}

(Received 12 May 2003; accepted 28 July 2003)

\begin{abstract}
We found that a focused laser can generate microscopic phase separation in an oil/water system. An oil droplet emerges and grows at the focus of the laser in a water-rich homogeneous medium. In contrast, in an oil-rich homogeneous phase, water droplets spring out in a successive manner from the focus of the laser, move away, and disappear in the surroundings, forming a flower-like pattern. The mechanism of this dynamic phase separation is discussed under the framework of the mean field theory. (C) 2003 American Institute of Physics. [DOI: 10.1063/1.1613795]
\end{abstract}

Liquid/liquid phase segregation in an oil/water system is a typical first-order phase transition. A homogeneous phase separates into two different phases with a change in a control parameter such as temperature. After passing through the binodal line from the homogeneous phase, the interface area in phase separation tends to decrease toward a minimum to reduce the penalty due to interfacial energy. This means that phase separation on a microscopic scale is usually unstable and coarsening is unavoidable. The most popular method for generating microscopic phase separation is to add surfaceactive agents to the oil/water system. Depending on the relative sizes of the head and tail of the surfactant molecules, and also their lateral interaction, a characteristic length scale can be introduced to the system, i.e., microscopic segregation is generated. ${ }^{1}$ In the present report, we show that a focused laser can generate microscopic phase separation in the absence of any surface-active agents. It has been well established that a focused laser can act as optical tweezers, generating attractive potential due to dielectric interaction. ${ }^{2-5}$ It has been shown that, at room temperature, the optical attractive force can overcome thermal fluctuation for a macromolecular system. Successful experiments on the optical trapping of an aggregate of synthetic polymer and a single DNA molecule with a compact folded conformation have been reported. ${ }^{6,7}$ There has been no report on optical trapping for small molecules below a molecular weight of several hundred Daltons in solution at room temperature, around $300 \mathrm{~K}$.

We have adapted a system with water and triethylamine as an oil/water system. This system exhibits the phase diagram shown in Fig. 1; phase segregation is generated with an increase in temperature at a fixed composition. Sample solutions were prepared by mixing pure water and triethylamine in a volume ratio of $1: 1$, and the mixture was allowed to stand for more than $1 \mathrm{~h}$ until equilibrium was reached. From the phase-separated solutions, the water-rich phase or oil-rich phase was transferred to a thin chamber $(10 \mathrm{~mm} \times 10 \mathrm{~mm}$ $\times 200 \mu \mathrm{m})$. We used two different laser sources: a Ti:sapphire laser (Millennia 3900S, Spectra Physics) and a yttrium-aluminum-garnet (YAG) laser (Millennia IR, Spec-

\footnotetext{
${ }^{a}$ Present address: The Deepstar group, Japan Marine Science and Technology Center, 2-15 Natsushima-cho, Yokosuka 237-0061, Japan.

b) Author to whom correspondence should be addressed; electronic mail: yoshikaw@scphys.kyoto-u.ac.jp
}

tra Physics). A converged laser was passed through an oilimmersed objective lens $(100 \times$, numerical aperture $=1.3)$ with an inverted microscope (TE-300, Nikon). Experiments were carried out at room temperature $(297 \pm 3 \mathrm{~K})$.

Figure 2(a) shows a spatiotemporal image of the emergence/nucleation and successive growth of an oil droplet at the focus of the laser $(\lambda=1064 \mathrm{~nm})$ in homogeneous medium rich in water at a laser power of $P=1.5 \mathrm{~W}$, where the actual shapes of the droplets are given at the bottom. Just after the start of irradiation at a certain place in the homogeneous solution, a small droplet appeared, and after $\sim 80 \mathrm{~s}$, the droplet reached a constant diameter of $\approx 49 \mu \mathrm{m}$. We found that such stable trapping is observed above $P$ $\approx 1.4 \mathrm{~W}$ [Fig. 2(b)]. When $1.2 \mathrm{~W}<P<1.4 \mathrm{~W}$, small oil droplets $(d \sim 5 \mu \mathrm{m})$ were generated, floated outward from the focus, and then disappeared into the homogeneous phase, in a successive manner. When $P<1.1 \mathrm{~W}$, no droplets were formed. We performed a similar experiment in which $\mathrm{H}_{2} \mathrm{O}$ was replaced by $\mathrm{D}_{2} \mathrm{O}$. It is known that the absorption of the length $(\lambda=1064 \mathrm{~nm})$ in $\mathrm{D}_{2} \mathrm{O}$ is less than $1 / 10$ of that in $\mathrm{H}_{2} \mathrm{O} .{ }^{8}$ We have confirmed that there is no essential difference in the experimental trends between $\mathrm{H}_{2} \mathrm{O}$ and $\mathrm{D}_{2} \mathrm{O}$, suggesting that the heating effect of the laser has little or no effect on the induction of microscopic phase separation.

Figure 3 shows spatiotemporal images of when the oilrich phase is irradiated with a laser $(\lambda=800 \mathrm{~nm})$. Soon after

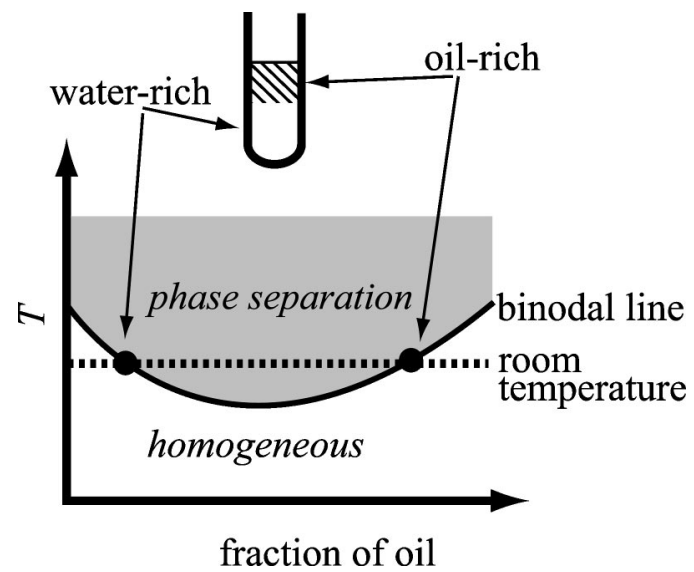

FIG. 1. Schematic phase diagram of the chosen water/oil system. The horizontal axis is the fraction of oil and the vertical axis is temperature. At room temperature, this system is separated into two phases, a water-rich phase and an oil-rich phase. 
(a)

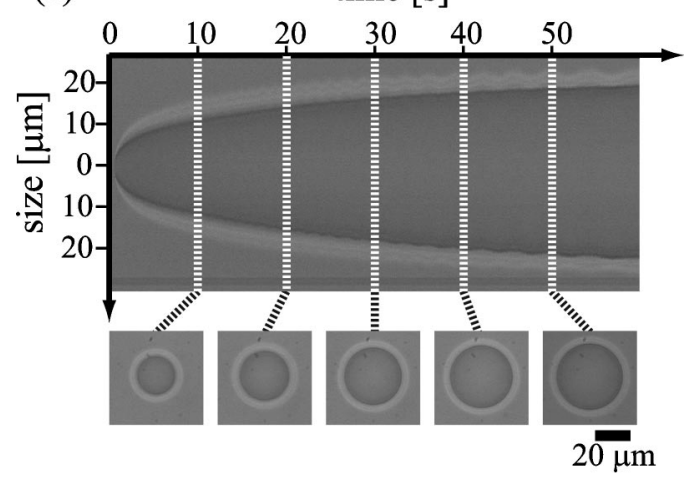

(b)

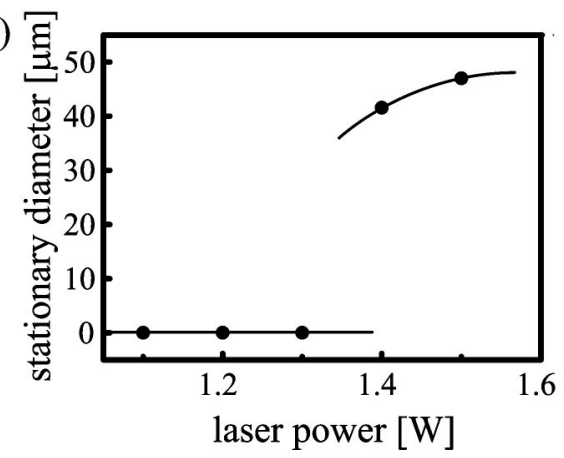

FIG. 2. (a) Spatiotemporal image of the nucleation and growth of an oil droplet at the focus of a YAG laser $(\lambda=1064 \mathrm{~nm})$ in water-rich medium at a laser power of $P=1.5 \mathrm{~W}$, reconstructed from time-successive video frames. Actual pictures of the droplets are shown at the bottom. (b) Dependence of the size of a stationary oil droplet on laser power. When the laser power is above $1.2 \mathrm{~W}$, small oil droplets were generated. Above $1.4 \mathrm{~W}$, small oil droplets were trapped by the attractive laser force and grew to a stable size.

the start of irradiation, small water droplets were generated, and these droplets moved away from the focus. During irradiation, small water droplets emerged continuously. The emerged droplets moved away from the focus and disappeared in the surroundings. We confirmed that essentially the same dynamical pattern of the droplets was observed with either $\mathrm{H}_{2} \mathrm{O}$ or $\mathrm{D}_{2} \mathrm{O}$, or with a laser wavelength of between 800 and $1064 \mathrm{~nm}$.

Based on the results given in Figs. 2 and 3, it is clear that a focused laser can induce microscopic phase separation in a

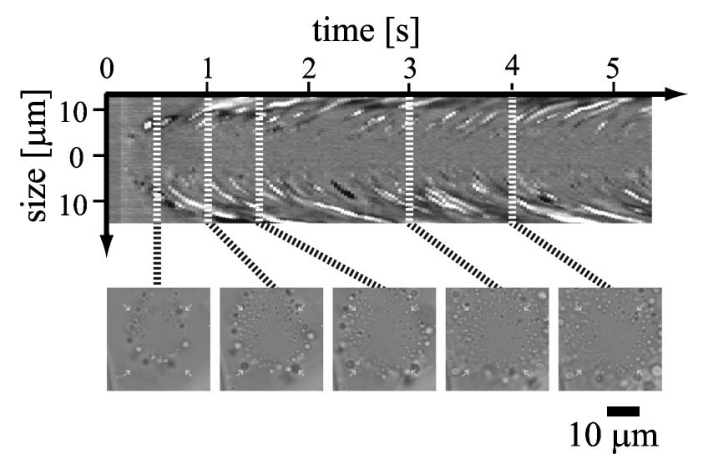

FIG. 3. Spatiotemporal image of the dynamical pattern of water droplets at the focus of a Ti:sapphire laser ( $\lambda=800 \mathrm{~nm}$ : variable) in oil-rich medium at a laser power of $P=2 \mathrm{~W}$, reconstructed from time-successive video frames. Actual pictures of the droplets are shown at the bottom. The droplets were repelled from the laser focus, reached the region beyond the optical potential, and then disappeared. This generation and annihilation of water droplets continued during irradiation with the laser.

Downloaded 06 Mar 2008 to 130.54.110.22. Redistribution subject to AIP license or copyright; see http://apl.aip.org/apl/copyright.j homogeneous medium. Depending on the composition of the solution, the generated droplet is stably trapped or is escaped from the focus. It has been well established that materials with high refractivity and polarizability are attracted to the focus. When the generated droplet is oil rich, it is trapped due to the attractive potential (Fig. 2). In contrast, when the generated droplet is water rich, it is repelled from the focus (Fig. 3) ${ }^{9}{ }^{910}$ The repelled droplets reach a region away from the optical potential, and disappear into the medium due to the loss of stability of the phase-separated state. Such generation and annihilation of the water droplet continue during constant irradiation with the continuous-wave laser.

We will now discuss the attractive potential of a laser in comparison with its thermal energy. In general, the optical force can be described in terms of the contribution of two components: the Rayleigh and Mie regimes. Since the initial appearance, or nucleation, of a droplet is the essential process to cause the microscopic phase separation, we will focus our discussion on the Rayleigh regime, where the attractive potential is given as

$$
\Delta U=-\frac{\kappa-1}{\kappa+2} \frac{3}{N} \cdot \varepsilon_{0} \frac{E^{2}}{2}=-\frac{3 M(\kappa-1)}{N_{A} d(\kappa+2)} \cdot \varepsilon_{0} \frac{E^{2}}{2},
$$

from Clausius-Mosotti's relation. ${ }^{11} N$ : number of molecules per volume, $N_{A}$ : Avogadro number, $\kappa$ : relative dielectric constant, $M$ : molecular weight, $d$ : density, $E^{2}$ : intensity of electro magnetic field. Using $\kappa=2.4, \quad M=101.2, d$ $=0.73 \mathrm{~g} / \mathrm{cm}^{3}$ for triethylamine and $\varepsilon_{0} E^{2} / 2=3.3 \times 10^{3} \mathrm{~J} / \mathrm{m}^{3}$ (In our experiments, the laser power, $P$, is $\sim 1 \mathrm{~W}$, and the characteristic scale of the focus, $\delta$, is $\sim 1 \mu \mathrm{m}$. Thus, the energy density of the electromagnetic field is estimated as $\varepsilon_{0} E^{2} / 2=P / c \delta^{2}=3.3 \times 10^{3} \mathrm{~J} / \mathrm{m}^{3}$.), we can estimate $|\Delta U|$ $\sim 7 \times 10^{-25} \mathrm{~J} \sim 10^{-4} k_{B} T$, where $T=300 \mathrm{~K}$. It is obvious that the attractive optical potential on a single oil molecule is much smaller than the thermal energy. This estimation of the attractive potential is consistent with previous studies on optical trapping, ${ }^{2-5}$ since only giant molecules can be trapped in solutions around room temperature. For example, a polymer chain with a degree of polymerization of $10^{4}$ from monomers of $10^{2}$ Daltons receives an attractive energy on the order of $k_{B} T$. In the present study, we have shown the stable optical trapping of a microscopic oil droplet with small molecules, by adopting an experimental condition near the binodal line (see Fig. 1).

We will now briefly discuss the generation and growth of an oil droplet, as in Fig. 2, under the framework of the mean field theory. ${ }^{1}$ We introduce an order parameter, $\eta(\mathbf{r})$ depending on the position $\mathbf{r}$, as a normalized parameter for the fraction of oil: $\eta(\mathbf{r})=1$ in the oil-rich phase, $\eta(\mathbf{r})=0$ in the water-rich phase. $f[\eta(r)]$ is a local free energy that depends only on the composition, with two local minima at $\eta=0$ and 1. We assume that the free energy under laser irradiation can be described by the product of the order parameter and $U(\mathbf{r})$, where $U(\mathbf{r})$ is a function providing attractive effect on the oil phase toward the laser focus owing to the difference of the permittivity. The total free energy $F[\eta(\mathbf{r})]$ is given as follows: ${ }^{1}$

$$
F[\eta(\mathbf{r})]=\int_{d} d \mathbf{r}\left\{f[\eta(\mathbf{r})]+\eta(\mathbf{r}) U(\mathbf{r})+c|\nabla \eta(\mathbf{r})|^{2}\right\},
$$




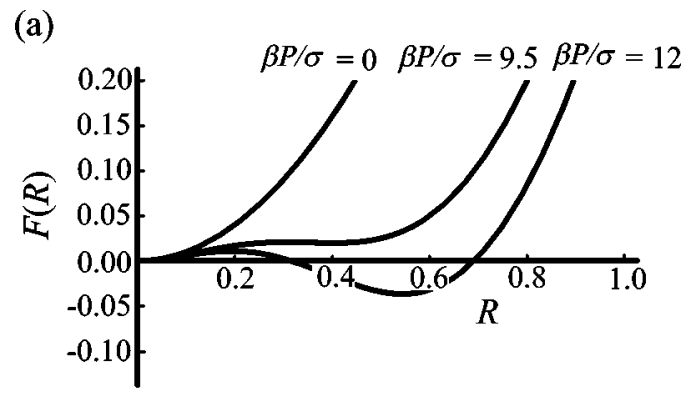

(b)

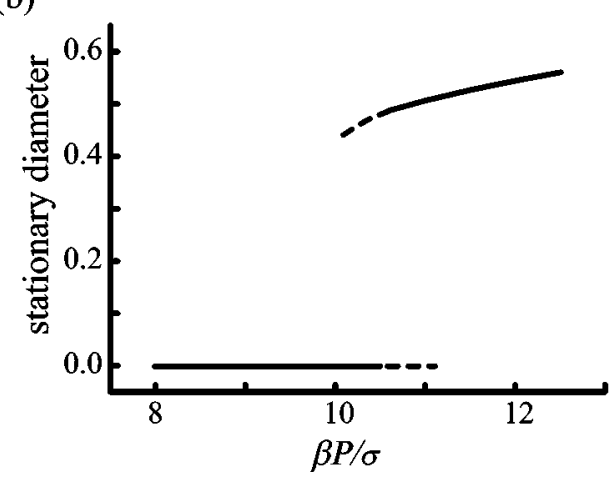

FIG. 4. (a) Theoretical free energy profile as a function of the laser power, $\beta P / \sigma$. When $\beta P / \sigma>10.6$, a global minimum appears at a certain size, $R$, of the droplet. (b) Droplet size as a function of laser power.

where $c$ is a constant related to the interfacial energy. For simplicity, the laser profile is assumed to be spherically symmetrical with a Gaussian distribution

$$
U(\mathbf{r})=-\beta P \exp \left(-\frac{r^{2}}{\omega^{2}}\right) \equiv U(r)
$$

where $P$ is the laser power, $\beta$ is a coupling constant, $\omega$ is the characteristic width of the laser potential, $r$ is the distance from the origin, or the center of the laser focus. The system exhibits excess energy in proportion to the interfacial area. This proportional constant is the interfacial tension $\sigma$ [The surface tension $\sigma$ is proportional to $c^{3 / 2}$ (see Ref. 1).]. We assume that $\eta(\mathbf{r})$ has spherical symmetry, and that $\eta=1$ and 0 at the focus and in the surroundings, respectively. This corresponds to a droplet of the oil-rich phase formed at the focus. We can write down the total energy only as a function of $R$, the radius of the droplet

$$
\begin{aligned}
F(R) & =\int_{0}^{R} 4 \pi r^{2} U(r) d r+4 \pi \sigma R^{2} \\
& =4 \pi \sigma\left[-\frac{\beta P}{\sigma} \int_{0}^{R} r^{2} \exp \left(-\frac{r^{2}}{\omega^{2}}\right) d r+R^{2}\right] .
\end{aligned}
$$

Changing the value of the parameter $\beta P / \sigma$, we can obtain various free energy profiles for $F(R)$ [Fig. 4(a)]. When $\beta P / \sigma>9.4$, this model has a local minimum value. Above $\beta P / \sigma \approx 10.6$, this local minimum becomes a global minimum value. This free energy minimum corresponds to a stationary state [Fig. 4(b)]. Figure 4(b) suggests a discrete transition in the generation of a droplet, in accordance with the experimental trend seen in Fig. 2. A similar argument will hold for the generation of a dynamical flower pattern, except for the inversion of the optical force that attracts the droplet generated around the focus.

In summary, it is clear that a laser field can generate microscopic phase separation. Since the potential profile of the laser is determined by the spatial distribution of optical power, it may be possible to use a laser field to generate and control phase transition at a microscopic scale. The most interesting finding in the present study may be the observation of the successive generation and annihilation of droplets in a homogeneous environment. Further theoretical consideration is necessary to interpret this dynamical behavior of the transition.

This research was supported in part by a Grant-in-Aid from the Ministry of Education, Science, Sports and Culture of Japan, and by a "Ground-based Research Announcement for Space Utilization" promoted by the Japan Space Forum.

${ }^{1}$ S. A. Safran, Statistical Thermodynamics of Surfaces, Interfaces, and Membranes (Perseus, Reading, MA, 1994).

${ }^{2}$ A. Ashkin, J. M. Dziedzic, J. E. Bjorkholm, and S. Chu, Opt. Lett. 11, 288 (1986).

${ }^{3}$ K. Visscher and G. J. Brakenhoff, Optik (Stuttgart) 89, 174 (1992).

${ }^{4}$ Y. R. Shen, The Principles of Nonlinear Optics (Wiley, New York, 1984).

${ }^{5}$ K. Svoboda and S. M. Block, Annu. Rev. Biophys. Biomol. Struct. 23, 247 (1994).

${ }^{6}$ P. Borowicz, J. Hotta, K. Sasaki, and H. Masuhara, J. Phys. Chem. B 101, 5900 (1997).

${ }^{7}$ S. Katsura, K. Hirano, Y. Matsuzawa, K. Yoshikawa, and A. Mizuno, Nucleic Acids Res. 26, 4943 (1998).

${ }^{8}$ M. Ishikawa, H. Misawa, N. Kitamura, R. Fujisawa, and H. Masuhara, Bull. Chem. Soc. Jpn. 69, 59 (1996).

${ }^{9}$ K. Sasaki, M. Koshioka, H. Misawa, N. Kitamura, and H. Masuhara, Appl. Phys. Lett. 60, 807 (1992).

${ }^{10}$ S. Katsura, A. Yamaguchi, H. Inami, S. Matsuura, K. Hirano, and A. Mizuno, Electrophoresis 22, 289 (2001).

${ }^{11}$ C. J. F. Böttcher, Theory of Electric Polarization (Elsevier, Amsterdam, 1952). 